\title{
Progress of plasma wakefield self-modulation experiments at FACET
}

\author{
E. Adli ${ }^{\mathrm{a}, \mathrm{b}}$, V. K. Berglyd Olsen ${ }^{\mathrm{a}, \mathrm{b}}$, C. A. Lindstrøm ${ }^{\mathrm{a}, \mathrm{b}}$, P. Mugglic ${ }^{\mathrm{c}}$ O. Reimann ${ }^{\mathrm{c}}$, J.M. Vieira ${ }^{\text {d }}$, L.D. Amorim ${ }^{\mathrm{d}}$, C. I. Clarke $^{\mathrm{b}}$, S. J. \\ Gessner $^{\mathrm{b}}$, S. Z. Green ${ }^{\mathrm{b}}$, M.J. Hogan ${ }^{\mathrm{b}}$, M. D. Litos ${ }^{\mathrm{b}}$, B. D. O’Shea ${ }^{\mathrm{b}}$, V. Yakimenko ${ }^{\mathrm{b}}$, C. Clayton ${ }^{\mathrm{e}}$, K. A. Marsh ${ }^{\mathrm{e}}$, W. B. Mori ${ }^{\mathrm{e}}$, C. $^{\circ}$ \\ Joshi $^{\mathrm{e}}$, N. Vafaei-Najafabadi ${ }^{\mathrm{e}}, \mathrm{O}$. Williams ${ }^{\mathrm{e}}$ \\ ${ }^{a}$ Department of Physics, University of Oslo, N-0316 Oslo, Norway \\ ${ }^{b}$ SLAC National Accelerator Laboratory, 2575 Sand Hill Road, Menlo Park, California 94025, USA \\ ${ }^{c}$ Max Planck Institute for Physics, Munich, Germany \\ ${ }^{d}$ GoLP/Instituto de Plasmas e Fusão Nuclear, Instituto Superior Téchnico, Universidade de Lisboa, 1049-001 Lisbon, Portugal \\ ${ }^{e}$ University of California Los Angeles, Los Angeles, CA 90095, USA
}

\begin{abstract}
Simulations and theory predict that long electron and positron beams may under favorable conditions self-modulate in plasmas. We report on the progress of experiments studying the self-modulation instability in plasma wakefield experiments at FACET. The experimental results obtained so far, while not being fully conclusive, appear to be consistent with the presence of the selfmodulation instability.
\end{abstract}

Keywords: lepton beams, plasma wakefield, self modulation instability, FACET User Facility

Wednesday $17^{\text {th }}$ February, 2016, 10:24

\section{Introduction}

Plasma wakefield accelerators [1] have the potential to provide very strong accelerating fields which can be used for particle acceleration. If the particle driver density, $n_{b}$, is much less than the plasma density, $n_{0}$, the plasma response will be in the linear regime [2, 3] and the accelerating fields relatively weak. It has been shown in simulations that a relativistic beam, long with respect to the plasma skin depth $k_{p} \sigma_{z} \gg 1$, and with initial low density $n_{b} \ll n_{0}$, may evolve to drive relatively stronger fields in the plasma, due to the self-modulation instability (SMI) [4]. $\sigma_{z}$ is here the bunch length, and $k_{p}$ is the cold plasma skin ${ }_{28}$ depth. The SMI radially modulates the beam such that micro- ${ }_{29}$ bunches with density much higher than $n_{b}$ are generated, with ${ }_{30}$ a spatial frequency close to the plasma electron wavelength, ${ }_{31}$ $\lambda_{p}=\frac{2 \pi}{k_{p}}$. The wake of the micro-bunches builds up coherently, ${ }_{32}$ leading to much stronger fields in the plasma than those set up ${ }_{33}$ by the initial beam. The AWAKE experiment at CERN [5, 6] ${ }_{34}$ will investigate the SMI with long proton bunches, and eventu- 35 ally exploit the SMI proton wake to accelerate electron bunches ${ }_{36}$ to high energy. Simulations, performed using the code OSIRIS ${ }_{37}$ [7], further show that also long electron and positron bunches 38 may experience the SMI [8, 9]. We have performed experi- ${ }_{39}$ ments at FACET [10] where electron and positron beams with 40 $k_{p} \sigma_{z} \gg 1$ have been sent into pre-formed plasmas, with the aim ${ }_{41}$ of observing evidence for the occurrence of the SMI.

Email address: Erik.Adli@fys.uio.no (E. Adli)

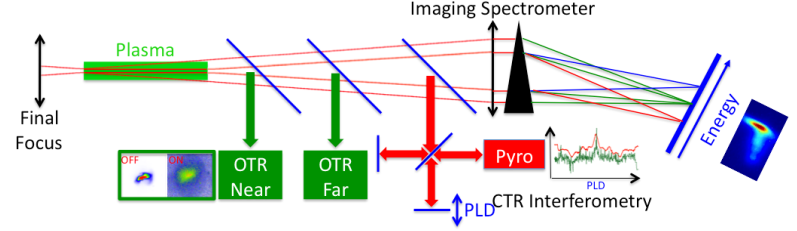

Figure 1: In the experiment, long $\left(k_{p} \sigma_{z} \gg 1\right)$ electron and positron bunches are sent into meter long plasmas generated from lithium vapour or noble gases. Signatures for the self-modulation instability are studied with three types of diagnostics; a spectrometer, transverse profile monitors (OTRs) and an interferometer.

\section{Experimental set-up}

Figure 1 shows the experimental set-up. FACET provides electron and positron beams with $3 \mathrm{nC}$ of charge and $20.3 \mathrm{GeV}$ energy [10]. Two plasma sources have been used; a laserionized lithium heat pipe oven [13] and tubes with laser-ionized hydrogen or argon gas. The plasma densities studied range from $8 \times 10^{16} \mathrm{~cm}^{-3}$ to $7 \times 10^{17} \mathrm{~cm}^{-3}$. In later discussions we will state which beam-plasma configuration was used. The final focus system of FACET provides focusing down to beta functions $\left(\beta^{*}\right)$ of a few $\mathrm{cm}$. We note that such focusing is not strong enough to match the beam into a plasma cavity in the blow out regime [14]; the matched beta function for a $20 \mathrm{GeV}$ beam in a plasma cavity with ion density of $8 \times 10^{16} \mathrm{~cm}^{-3}$ is $5 \mathrm{~mm}$. Typical electron beam spot sizes at the plasma entrance were measured to about $40 \mu \mathrm{m}$ rms, with some variation from day to day. Typical bunch lengths were measured to about $1.5 \mathrm{~mm}$ FWHM. Examples of the bunch charge profiles measured are given in Figure 2. The shot to shot variation of the measured bunch lengths is about $1 \%$ for the measurement series shown. 


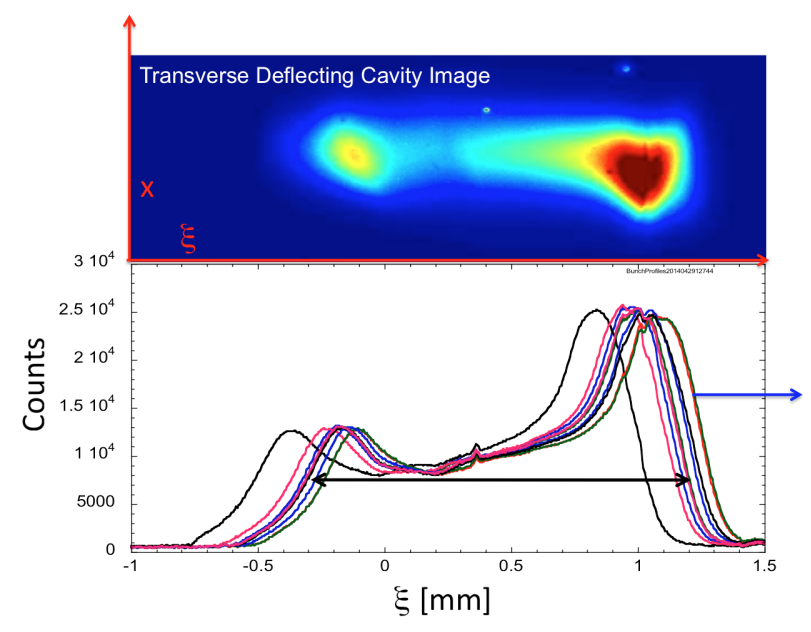

Figure 2: Bottom inset: example bunch profiles, as measured using a transverse deflecting structure. The measured bunch length is about $1.5 \mathrm{~mm}$, with a twohump profile. The bunch is traveling towards the right. Top inset : example of the $2 D$ beam profile measured using a transverse deflecting structure.

A single-shot measurement of bunch modulation after pass- ${ }_{81}$ ing through the plasma would be ideal for demonstrating the ${ }_{82}$ SMI. However, the expected modulation frequency is of the or- ${ }_{83}$ der of $100 \mu \mathrm{m}$, which is too small to be resolved by the fastest ${ }_{84}$ streak cameras available [15]. Three other types of diagnostics were used to look for signatures of the SMI; a spectrometer, transverse profile monitors and an interferometer.

The FACET imaging spectrometer consists of a dipole dispersing the beam according to energy, plus two quadrupoles ${ }_{86}$ which image (focus) particles of selectable energy. The spec- ${ }_{87}$ trometer images are recorded by Lanex and Cherenkov profile ${ }_{88}$ monitors [16] with an energy resolution better than $100 \mathrm{MeV}$. A signature for self-modulation would be the presence of fields ${ }_{90}$ in the plasma which are stronger than expected than if the beam ${ }_{91}$ had not self-modulated.

Transverse profile monitors based on optical transition radi- ${ }_{93}$ ation (OTR) are located downstream of the plasma. The "near" 94 OTR is $1.8 \mathrm{~m}$ downstream of the plasma exit, while the "far" is $3.0 \mathrm{~m}$ downstream. The SMI is expected to expel radially 96 a significant fraction of the beam. Preliminary simulation re- ${ }_{97}$ sults [7, 11] indicate that a few $10 \mathrm{~s}$ of $\mathrm{cm}$ into the plasma, outer ${ }_{98}$ parts of the beam have picked up divergence of the order of one ${ }_{99}$ mrad. After $1.3 \mathrm{~m}$ of plasma propagation, roughly half of the $\mathrm{e}_{100}$ initial charge has a large divergence and is located at large radii ${ }_{101}$ $(\mathrm{r} \gtrsim 500 \mu \mathrm{m})$. A signature for self-modulation would therefore ${ }_{102}$ be the presence of charge at large radial positions.

A Michelson interferometer with pyro detectors is installed ${ }_{104}$ in order to pick up coherent transition radiation generated by ${ }_{105}$ the beam as it passes through a titanium foil [12]. By scanning ${ }_{106}$ the interferometer arm, an auto-correlation signal of the incom-107 ing radiation is produced. Assuming the radiation amplitude ${ }_{108}$ is proportional to the charge squared for a given longitudinal ${ }_{109}$ slice of the beam, the auto-correlation signal will contain har-110 monics of eventual modulations present along the beam. Since ${ }_{111}$ the SMI is expected to modulate the beam density with wave-112
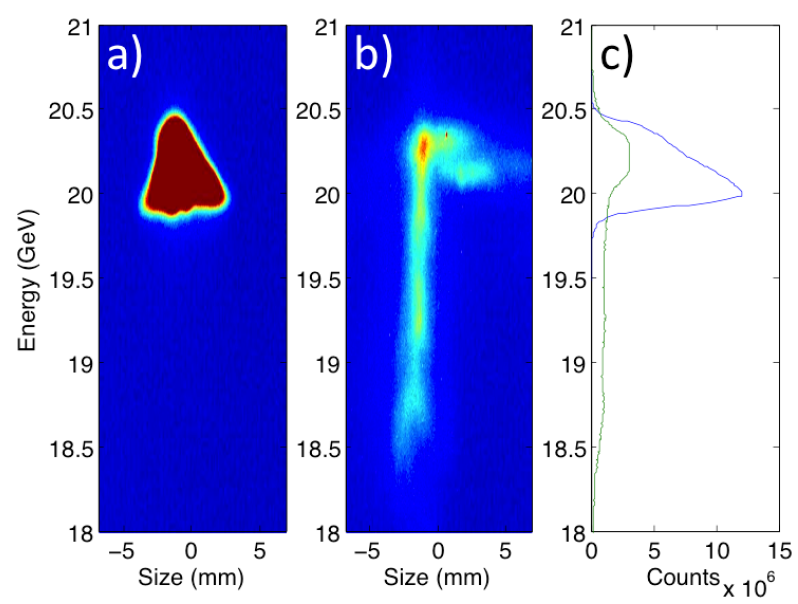

Figure 3: Electron-lithium results: a) The measured energy spectrum of the electron beam not passing through plasma. The beam entering the plasma has an energy spread of a few \%. b) The measured energy spectrum of the electron beam having passed through the plasma. Particles are decelerated by up to almost $2 \mathrm{GeV} . \mathrm{c}$ ) The projection of the two spectra.

length $\sim \lambda_{p}$ [4], and assuming the radially expelled charge does not contribute to the radiation generated, a signature for selfmodulation would be the presence of spectral content at the plasma wavelength in the auto-correlation signal.

\section{Experimental results}

First, we report results from electron beams in laser-ionized lithium with a plasma length of about $1.3 \mathrm{~m} \mathrm{FWHM}$ and plasma density of $n_{0}=8 \times 10^{16} \mathrm{~cm}^{-3}$. As the ionization energy for lithium is relatively low $(5.4 \mathrm{eV})$, the $10 \mathrm{TW} \sim 500 \mathrm{~mJ}$ FACET laser is expected to ionize a plasma channel of $\sim 1 \mathrm{~mm}$ size [13], which is much larger than the bunch radius. Using the FACET spectrometer we observed an electron energy loss of up to 2 $\mathrm{GeV}$ in the plasma, corresponding to decelerating fields of almost $2 \mathrm{GV} / \mathrm{m}$. Figure 3 shows example experimental shots; a) shows the energy spectrum when the beam has not passed through plasma (the ionization laser is off); b) shows the energy spectrum when the beam has passed through the lithium plasma. Compared to predictions in [8, 9] the results are reasonably consistent with a beam that has self-modulated. As further comparison, we make an energy loss estimate by calculating the wakefields originating from the measured bunch profile (Figure 2] using linear theory [3], according to the method described in [17]. The calculated longitudinal fields are no more than $100 \mathrm{MV} / \mathrm{m}$, much smaller than what is observed experimentally. This calculation implies that the charge density stays at the same order as the original density $\left(n_{b} \ll n_{0}\right)$. However, both simulations and experiments at FACET indicate that a bunch initially with low charge density $n_{b} \lesssim n_{0}$, may strongly self-focus in a plasma, increasing the charge density by several order of magnitude [19]. This mechanism could possibly lead to significantly stronger fields than what is estimated from linear theory, also in the absence of the SMI. 

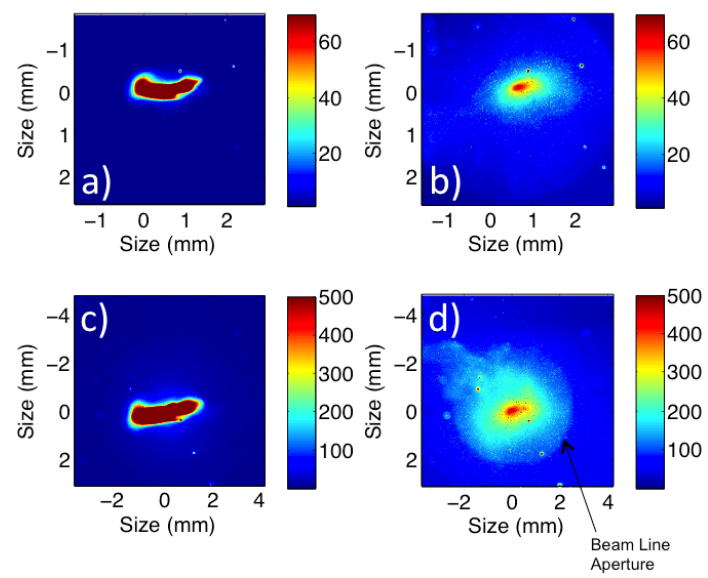

Figure 4: Electron-lithium results: a), c) The measured transverse beam profile of the electron beam not passing through plasma. b), d) The measured transverse beam profile of the electron beam having passed through plasma. A significant amount of the charge is located at large radii, for many shots up filling the available beam line aperture.

Significant halo formation was observed in the electronlithium experiments. This is illustrated in Figure 4 where a) and c) show the transverse beam profile of the beam, at the "near" OTR, when not passing through plasma, while b) and d) show the transverse beam profile of the beam when having passed through plasma. The halo formation in the latter case is qualitatively consistent with radial expulsion of a significant portion of the beam charge. However, other sources, like beamplasma mismatch, may also contribute to the formation of transverse beam halo. Similar halo formation has been observed in FACET experiments where short bunches $\left(k_{p} \sigma_{z} \sim 1\right)$ were sent into plasmas with similar densities as in the SMI experiments [18].

Figure 5 summarizes the results of an interferometry scan; a) shows the auto-correlation signal, as measured for the two cases of no plasma (upper, lighter curve) and plasma present (lower, darker curve). When the plasma is present in the beam path, the signal contains more high frequency components. The signal is also overall less strong, which is consistent with less radiating charge. b) shows the spectral content of the signal, found by performing a Fourier transform of a). The spectrum analysis confirms that with plasma present, a significant amount of high frequency content is present. For the plasma density $\left(8 \times 10^{16} \mathrm{~cm}^{-3}\right)$ the plasma wavelength is $\lambda_{p}=118 \mu \mathrm{m}$. We observe that spectral peaks close $\lambda_{p}$ and $\lambda_{p} / 2$ (the first harmonic) is present in the signal. However, other wavelengths not expected from a modulation at $\lambda_{p}$ are also present.

Figure 6 summarizes interferometry measurements for positron-argon SMI experiments, with plasma densities of $7.4 \times$ $10^{17} \mathrm{~cm}^{-3}, 2.4 \times 10^{17} \mathrm{~cm}^{-3}$ and $8 \times 10^{16} \mathrm{~cm}^{-3}$. Spectral content close the plasma wavelength was observed for the higher two densities. Peaks at about $95 \mu \mathrm{m}$, compared to the expected 115 $\mu \mathrm{m}$, was observed for the lower density. For this data as well, additional spectral content at wavelengths not expected from a modulation at the plasma density is present. To provide con-
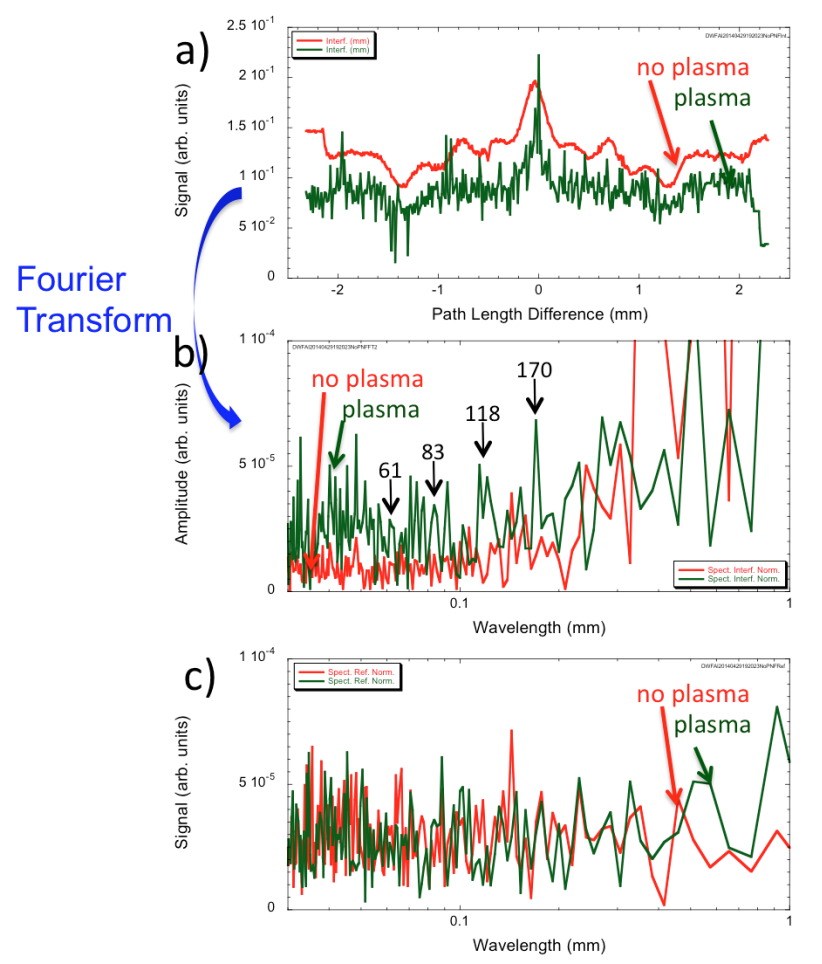

Figure 5: Electron-lithium results: a) Auto-correlation signal for the two cases of no plasma (upper, lighter curve) and plasma (lower, darker curve). When the plasma is present, the signal contains more high frequency components. $b$ ) the spectral content of the auto-correlation signal. The plasma wavelength is $\lambda_{p}=$ $118 \mu \mathrm{m}$ for the plasma density used $\left(8 \times 10^{16} \mathrm{~cm}^{-3}\right)$. Spectral peaks close $\lambda_{p}$ and $\lambda_{p} / 2$ (the first harmonic) is present in the signal. However, other wavelengths not expected from a modulation at $\lambda_{p}$ is also present. c) the spectral content of a reference signal that picks up the radiation entering the interferometer, without being auto-correlated. The reference signal does not contain strong peaks related to $\lambda_{p}$. 


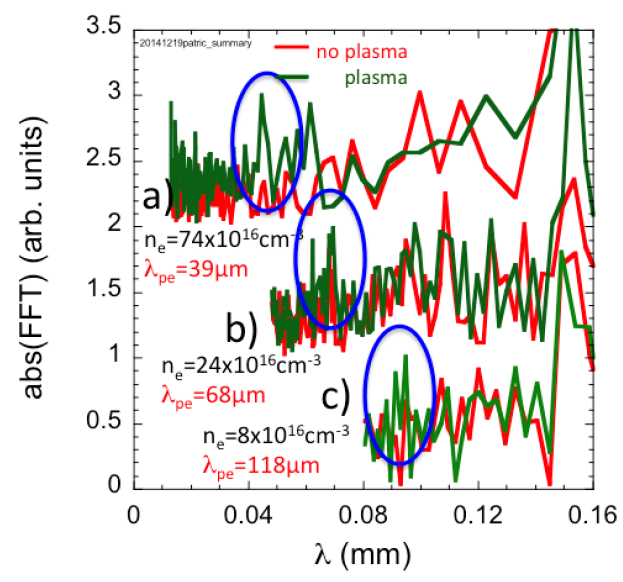

Figure 6: Positron-argon results: spectral content of auto-correlation measure- ${ }^{197}$ ments for three different plasma densities, $7.4 \times 10^{17} \mathrm{~cm}^{-3}, 2.4 \times 10^{17} \mathrm{~cm}^{-3}$ and $^{198}$ $8 \times 10^{16} \mathrm{~cm}^{-3}$. Spectral content close to $\lambda_{p}$ is observed for the higher two den- ${ }_{200}^{199}$ sities. [16
[2] T. Katsouleas et al., Particle Accelerators, vol. 22, 1 (1987), p. 81

[3] P. Chen et al., IEEE-TPS, PS-15, No. 2 (1987)

[4] N. Kumar, A. Pukhov and K. Lotov, Phys. Rev. Lett, 104, 255003 (2010)

[5] A. Caldwell et al., These proceedings (2015)

[6] E. Gschwendtner et al., These proceedings (2015)

[7] R. A. Fonseca et al., Lect. Notes Comput. Sci. 2331, 342 (2002), R. A. Fonseca, et al., Plasma Phys. Contr. F. 50, 124034 (2008).

[8] J.Vieira et al., Phys. Plasmas 19, 063105(2012)

[9] L.D. Amorim, J. Vieria, L.O. Silva and P. Muggli, In Proceedings of the 4th International Particle Accelerator Conference (2014), p. 1531

[10] M. J. Hogan et al., New J. Phys. 12, 055030 (2010)

[11] L.D. Amorim, priv. communicaiton.

[12] RadiaBeam Bunch Length Interferometer System, http://www . radiabeam.com/upload/1310535746BLIS_Manual_Interm.pdf

[13] S. Z. Green et al., Plasma Phys. Contr. Fusion 56, 084011 (2014).

[14] J.B. Rosenzweig, B. Breizman, T. Katsouleas, J.J. Su, Phys. Rev. A 44, R6189-R6192 (1991)

[15] M.D. Litos et al., In Proceedings of the 2011 Particle Accelerator Conference (2011), p. 568

[16] E. Adli, S.J. Gessner, S. Corde, M.J. Hogan, H.H. Bjerke, Nucl. Instrum. Methods Phys. Res. A 783, 35-42 (2015).

[17] P. Muggli et al., In Proceedings of the 5th International Particle Accelerator Conference (2015), p. 1476

[18] The FACET E200 experiment. Private communication.

[19] S. Corde et al., Submitted for publication. clusive results it is desired that the spectra show significantly stronger signals at the expected wavelengths than elsewhere. Alternatively, an explanation for the unexpected peaks in the spectra would be needed. Thus, either improved data or improved analysis is required to bring the experimental results to conclusion.

Electron-hydrogen SMI experiments were performed in order to provide more statistics and improved data. The progress of these experiments were impeded by too narrow plasma channel formation due to a combination of less-than-nominal laser power, and the relatively high ionization potential for hydrogen $(13.6 \mathrm{eV})$. Due to shot to shot beam jitter, the large majority of the experimental shots would not pass cleanly through the plasma channel.

\section{Conclusions}

We have investigated the development of the self-modulation instability (SMI) in experiments at FACET. The measurements of a long electron bunch having interacted with a lithium plasma are consistent with a bunch that has self-modulated. However, evidence for SMI is not fully conclusive at the time of writing. More data, or possibly improved analysis, is required to arrive at a firm conclusion.

\section{Acknowledgments}

Work at SLAC was supported by DOE contract number DEAC02-76SF00515 and also through the Research Council of Norway. Work at UCLA was supported by DOE grant number. DE-SC0010064 and the NSF grant number PHY-1415386.

\section{References}

[1] P. Chen, J. Dawson, R. Huff and T. Katsouleas, Phys. Rev. Lett. 54, 693(1985) 